\title{
Relationships between Acculturative Stress, Depression, and Quality of Life on in North Korean Refugees Living in South Korea
}

\section{Ji Hyun $\mathrm{Oh}^{*}$}

Department of Nursing, Daejeon University, South Korea

*Corresponding author: Ji Hyun Oh, Assistant Professor, Department of Nursing, Daejeon University, South Korea, Tel: 82-10-3002-4638; E-mail: grape0123@daum.net

Rec date: Sep 03, 2015; Acc date: Nov 09, 2015; Pub date: Nov 11, 2015

Copyright: $(2015$ Hyun Oh J. This is an open-access article distributed under the terms of the Creative Commons Attribution License, which permits unrestricted use, distribution, and reproduction in any medium, provided the original author and source are credited.

Abstract
Purpose
The aim of this study was to evaluate acculturative stress, depression, and quality of life, and investigate the
relationship between these factors in North Korean refugees who settled in South Korea.
Methods
A total of 232 North Korean refugees residing in South Korea participated in the study. Participants completed a
series of questionnaires, including measures of acculturative stress, depression, and quality of life. Data were
analyzed using SPSS WIN 20.0.
Results
The results showed that Acculturative stress, depression, and quality of life were interrelated. In our sample,
$40.9 \%$ of participants scored $\geq 16$ on the BDI-II, and the mean depression score was 15.42 (range $0-55$ ), which
indicates mild depressive symptoms. The mean total acculturative stress score was 2.88 (SD=0.02). The mean total
QOL total score was 3.02 (SD=0.03). The most powerful predictor of quality of life was depression in North
Korean refugees. Conclusion: The results suggest that acculturative stress is a factor in preventing depression and
promoting quality of life, especially mental health, in North Korean refugees.

Keywords: Acculturative stress; Depression; Quality of life; Refugee

\section{Introduction}

The number of North Korean refugees entering South Korea has greatly increased for economic reasons such as hunger, malnutrition, and fear of starvation. North Korea has suffered great economic hardship and famine. The number of North Korean defectors rose to 1,143 in $2002,1,896$ in 2004, 2,018 in 2006, and 2,805 in 2008. By the end of 2013, a total of 25,560 North Korean defectors had settled in South Korea [1]. In recent years, North Korean refugees have fled to neighboring nations such as China and third world countries [2] Hundreds of thousands of North Koreans have crossed the border to China in desperation to find the essential right to life that they were deprived by their government [3]. Many North Korean refugees experience psychological problems such as acculturative stress, depression, and post-traumatic stress disorder [4-6]. Problems that arise during the adaptation period can be aggravated by unsteady financial situations, social prejudice, and uncertainty about the future[3], as well as constant worry about government revenge against family members left in North Korea[4,6].

Acculturation is, defined as an individual's cultural learning process and the psychological change that occurs while adapting to an unfamiliar culture[7,8], and involves various forms of mutual accommodation that lead to longer-term psychological and sociocultural adaptations. Acculturation may contribute to risk for psychiatric problems because of the extended struggles to absorb the values, behaviors, and social norms of the host culture while simultaneously sustaining one's culture of origin [6]. Acculturative stress refers to tension or strain that is correlated with the acquisition of a second-culture that may result in negative physical or mental health effects [7]. As the number of North Korean refugees residing in South Korea has increased, their settlement has become an important issue in South Korean society and the international community [3]. Difficulties during the adaptation process are clearly related to mental health [6-9]. Chronic negative emotional experiences might be detrimental to the mental health of North Korean refugees [6] and exacerbate underlying psychological problems, rendering adaptation difficult at best [9]. Several previous studies have shown that many defectors experience acculturative stress and depression $[10,11]$. Refugees are at risk for mental health problems due to forced migration from their home countries and other traumatic experiences. Also, refugees may experience post-resettlement problems ranging from difficulty finding jobs and accessing services to cultural and linguistic isolation, all of which could increase risk for mental illness [12]. These psychosocial problems may impair quality of life and obstruct adaptation to life in a new culture $[13,14]$.

Quality of life (QOL) is a multidimensional concept that is associated with a person's health status [15]. Measuring patientperceived QOL has become one of the most important outcomes in 
health care and is used as a measure of effectiveness, both at the population and individual patient's levels [16]. Thus, the goals of this study were to investigate acculturative stress, depression, and QOL among North Korean refugees in South Korea, and to evaluate the relationship between mental health and QOL in this population.

\section{Methods}

\section{Participants}

The study was conducted from November to December 2013. Participants were selected using a snowball sampling method of individuals living in Seoul or the vicinity of the capital of South Korea. The snowball sampling method was used because North Korean refugees are often unwilling to make their identities known. The study population consisted of North Korean defectors over the age of 20 living in South Korea. The purpose and content of the study were explained to all 232 participants prior to administering the surveys. All participants were informed and assured of their right to refuse or withdraw from the study at any time. Institutional Review Board approval was received from the Ministry of Health and Welfare on Human Research (IRB No. P01-201309-SB-03-02) Instrument.

\section{Acculturative stress}

Acculturative stress was measured using the Korean version of the Acculturative Stress Scale developed by Sandhu and Asrabadi [17] and, translated into Korean by Lee [18]. The original scale consisted of 36 items from 7 subscales, including perceived discrimination (7 items), homesickness (4 items), perceived hate (5 items), fear (4 items), stress due to change/culture shock ( 3 items), guilt ( 2 items), and nonspecific concerns (10 items). However, 3 items were not applicable to our target population; thus, the final scale used here included 33 items. Each item was rated on a 5-point Likert scale ranging from strongly disagree (scored as 1) to strongly agree (scored as 5), with higher scores indicating higher levels of acculturative stress. Cronbach's alpha for this scale was .93 in a previous study [18] and .88 in this study.

\section{Depressive symptoms}

Depression was evaluated using the Korean version of the Beck Depression Inventory (BDI-II) [19] created, by Kim, Lee and Lee [20]. The BDI-II is a 21 -item, self-administered questionnaire designed to assess changes in and severity of depression during the previous week. Total BDI-II scores range from 0 to 63 , and each item is answered on a 4-point scale. The instrument developers established four groups of scores and classified them as the follows: "0-9: minimal or no depression; 10-15: mild depression; 16-23: moderate depression; and 24-63: severe depression". Higher scores indicate greater depressive symptoms. The reliability of this scale was .80 in a previous study [20]. Cranach's alpha for this scale was .87 in this study.

\section{Quality of life}

To measure QOL in North Korean refugees, a modified version of the QOL scale that included 47 questions [21] was used. The six domains evaluated by the QOL scale are physical condition and function, psychological condition, economic life, self-esteem, neighbor relations, and domestic relations. Items are rated on a 5-point Likert scale. Raw domain scores are converted to a 0-100 scale for ease of comparison with other data sets. Higher scores indicate better QOL.
The reliability of this scale was 0.94 in a previous study. Cronbach's alpha for this scale was 0.89 in this study.

\section{Statistical analysis}

Data were analyzed using SPSS, version 20.0. Descriptive statistics were used to assess the frequency distribution and sample characteristics. Pearson correlation coefficients between acculturative stress, depression, and QOL were calculated. Bivariate analyses were performed using t-tests or one-way analysis of variance (ANOVAs), as appropriate, to assess relationships between acculturative stress, depression, QOL, and independent socio-demographic characteristics. Scheffe's tests were performed to validate inter-group comparisons. Finally, a multiple regression analysis was conducted to determine the most predictive factors for QOL.

\section{Results}

\section{Participant demographic characteristics}

The results of the descriptive analysis for all variables are presented in Table 1. Table 1 shows the demographic characteristics of the North Korean refugees. There were 232 participants (65 male, 28.0\%; 167 female, $72.0 \%)$ who ranged in age from 23 to 84 years $(M=52.88$, $\mathrm{SD}=1.01$ ), most of whom were married. Approximately half of the participants (53.4\%) had low economic status, $57.8 \%$ had middle-high school education, $62.5 \%$ lived with family, $81.0 \%$ were unemployed. and $73.7 \%$ were protestant. Most participants reported that they were non-smokers (86.6\%), 67.7\% were non-drinkers, 59.1\% reported exercising, and $57.8 \%$ had lived in South Korea for 6-10 years. Participant's monthly income was below 500,000 Won (48.3\%), they had between 1 and 2 chronic disease, and lived in South Korea during an average of 99.63 months and lived in a third world country for an average of 33.93 months.

\begin{tabular}{|c|c|c|c|}
\hline Variables & Categories & $n(\%)$ & $M \pm S D$ \\
\hline \multirow{6}{*}{$\begin{array}{l}\text { Age(years) } \\
(M \pm S D, 52.88 \pm 1.01)\end{array}$} & $20-29$ & $13(5.6)$ & \\
\hline & $30-39$ & $35(15.1)$ & \\
\hline & $40-49$ & $58(25.0)$ & \\
\hline & $50-59$ & $42(18.1)$ & \\
\hline & $60-69$ & $32(13.8)$ & \\
\hline & $\geq 70$ & $52(22.4)$ & \\
\hline \multirow{2}{*}{ Gender } & Male & $65(28.0)$ & \\
\hline & Female & $167(72.0)$ & \\
\hline \multirow{4}{*}{ Marital status } & Unmarried & $35(15.1)$ & \\
\hline & Married & $124(53.4)$ & \\
\hline & Divorced & $29(12.5)$ & \\
\hline & Bereaved & $44(19.0)$ & \\
\hline \multirow{2}{*}{ Living with } & Alone & $87(37.5)$ & \\
\hline & Family & $145(62.5)$ & \\
\hline Religion & Protestant & $171(73.7)$ & \\
\hline
\end{tabular}


Citation: Hyun Oh J (2015) Relationships between Acculturative Stress, Depression, and Quality of Life on in North Korean Refugees Living in South Korea. J Health Edu Res Dev 3: 142. doi:10.4172/2380-5439.1000142

Page 3 of 8

\begin{tabular}{|c|c|c|}
\hline & Catholic & $2(0.9)$ \\
\hline & Buddhist & $10(4.3)$ \\
\hline & No religion & $49(21.1)$ \\
\hline \multirow{4}{*}{ Education } & None & $2(0.9)$ \\
\hline & Elementary school & $10(4.3)$ \\
\hline & Middle-High school & $134(57.8)$ \\
\hline & College & $86(37.1)$ \\
\hline \multirow{3}{*}{ Economic status } & High & $2(0.9)$ \\
\hline & Middle & $28(12.1)$ \\
\hline & Low & $202(87.1)$ \\
\hline \multirow{2}{*}{ Smoking } & Yes & $31(13.4)$ \\
\hline & No & $201(86.6)$ \\
\hline \multirow{2}{*}{ Drinking } & Yes & $75(32.3)$ \\
\hline & No & $157(67.7)$ \\
\hline \multirow{2}{*}{ Exercise } & Yes & 137(59.1) \\
\hline & No & $95(40.9)$ \\
\hline \multirow{2}{*}{ Occupation } & Yes & $44(19.0)$ \\
\hline & None & $188(81.0)$ \\
\hline \multirow{4}{*}{ Monthly income } & $\leq 500,000$ & $112(48.3)$ \\
\hline & $510,000-990,000$ & $87(37.5)$ \\
\hline & $100,000-149,000$ & $22(9.5)$ \\
\hline & $\geq 150,000$ & $11(4.7)$ \\
\hline \multirow{2}{*}{ Number of comorbidity } & 0 & $67(28.9)$ \\
\hline & $1-2$ & $121(52.2)$ \\
\hline
\end{tabular}

\begin{tabular}{|l|l|l|l|}
\hline \multirow{2}{*}{$(\mathrm{M} \pm \mathrm{SD}, 1.05 \pm 80)$ (years) } & $3-4$ & $38(16.3)$ & \\
\cline { 2 - 4 } & $\geq 5$ & $6(2.6)$ & \\
\hline Living in the other countries & months & & $33.93 \pm 1.96$ \\
\hline Duration in South Korea & months & & $99.63 \pm 2.52$ \\
\hline
\end{tabular}

Table 1: Participant Demographic Characteristics.

\section{Acculturative stress, depression, and QOL}

The means, standard deviations, and ranges for the study variables are presented in Table 2. At the time of the survey, the mean depression score was 15.42 (range 0-55), a score indicating mild depressive symptoms; $41.8 \%$ of respondents had no depressive symptoms, $17.2 \%$ had mild depressive symptoms, $12.9 \%$ had moderate depressive symptoms, and $28.0 \%$ had severe depressive symptoms. The mean total acculturative stress score was $2.88(\mathrm{SD}=0.02)$. The mean total QOL score was $3.02(\mathrm{SD}=0.03$; possible range $=1-5)$. For acculturative stress (possible range $=1-5$; higher scores indicate higher acculturative stress), homesickness ratings were highest, with a mean score of 3.66 $(\mathrm{SD}=0.04)$. The mean perceived discrimination score was 2.78 $(\mathrm{SD}=0.03)$, the mean perceived hate score was $2.12(\mathrm{SD}=0.02)$, the mean change/culture shock score was $2.72(\mathrm{SD}=0.03)$, the mean guilt score was $3.09(\mathrm{SD}=0.05)$, the mean fear score was $2.78(\mathrm{SD}=0.04)$, and the mean miscellaneous score was $3.06(\mathrm{SD}=0.03)$. For the $\mathrm{QOL}$ subscales (possible range $=1-5$; higher scores indicate better QOL), neighbor relation was reported as the most positive, with a mean score of $3.32(\mathrm{SD}=0.03)$. The mean economic life score was $3.08(\mathrm{SD}=0.03)$, the mean physical condition and function score was $2.93(\mathrm{SD}=0.03)$, the mean domestic relation score was $2.68(\mathrm{SD}=0.06)$, the mean psychological condition score was $2.94(\mathrm{SD}=0.04)$, and the mean selfesteem score was $3.19(\mathrm{SD}=0.04)$.

\begin{tabular}{|c|c|c|c|c|}
\hline Categories & Mean \pm SD & Actual range & Potential range & $n(\%)$ \\
\hline \multicolumn{5}{|l|}{ Acculturative stress } \\
\hline perceived discrimination & $2.78 \pm 0.03$ & $1.00-4.67$ & $1-5$ & \\
\hline homesickness & $3.66 \pm 0.04$ & $1.50-5.00$ & $1-5$ & \\
\hline perceived hate & $2.12 \pm 0.02$ & $0.80-3.20$ & $1-5$ & \\
\hline stress due to change/ culture shock items & $2.72 \pm 0.03$ & $1.00-5.00$ & $1-5$ & \\
\hline Guilt & $3.09 \pm 0.05$ & $1.00-5.00$ & $1-5$ & \\
\hline Fear & $2.78 \pm 0.04$ & $1.00-4.25$ & $1-5$ & \\
\hline miscellaneous & $3.06 \pm 0.03$ & $1.22-4.56$ & $1-5$ & \\
\hline Total & $2.88 \pm 0.02$ & $1.39-3.97$ & $1-5$ & \\
\hline \multicolumn{5}{|l|}{ Quality of life } \\
\hline economic life & $3.08 \pm 0.03$ & $1.09-5.00$ & $1-5$ & \\
\hline
\end{tabular}


Citation: Hyun Oh J (2015) Relationships between Acculturative Stress, Depression, and Quality of Life on in North Korean Refugees Living in South Korea. J Health Edu Res Dev 3: 142. doi:10.4172/2380-5439.1000142

Page 4 of 8

\begin{tabular}{|c|c|c|c|c|}
\hline physical condition/function & $2.93 \pm 0.03$ & $1.11-4.56$ & $1-5$ & \\
\hline domestic relation & $2.68 \pm 0.06$ & $0.33-5.00$ & $1-5$ & \\
\hline neighbor relation & $3.32 \pm 0.03$ & $1.00-5.00$ & $1-5$ & \\
\hline psychological condition & $2.94 \pm 0.04$ & $1.00-4.89$ & $1-5$ & \\
\hline self-esteem & $3.19 \pm 0.04$ & $1.00-4.88$ & $1-5$ & \\
\hline Total & $3.02 \pm 0.03$ & $1.11-4.79$ & & \\
\hline Depression & $15.42 \pm 0.91$ & $0-55$ & $0-63$ & \\
\hline minimal & & & & $97(41.8)$ \\
\hline Mild & & & & $40(17.2)$ \\
\hline moderate & & & & $30(12.9)$ \\
\hline severe & & & & $65(28.0)$ \\
\hline
\end{tabular}

Table 2: Acculturative Stress, Depression, and Quality of Life.

\section{Acculturative stress, depression, and QOL scores by participant's demographic characteristics}

Table 3 shows the differences in acculturative stress, depression, and QOL scores based on demographic characteristics. The analyses showed a significant difference in acculturative stress based on education $(\mathrm{F}=5.09, \mathrm{p}=0.002)$. There was a significant difference in QOL $(\mathrm{F}=6.09, \mathrm{p}=0.003)$ based on economic status. There was a significant difference in acculturative stress $(\mathrm{F}=2.09, \mathrm{p}=0.037)$, depression
$(\mathrm{F}=4.11, \mathrm{p}<0.001)$, and $\mathrm{QOL}(\mathrm{F}=-5.11, \mathrm{p}<0.001)$ based on exercise. There was a significant difference in depression $(\mathrm{F}=-2.11, \mathrm{p}=0.036)$ based on occupation. There was a significant difference in depression $(\mathrm{F}=4.32, \mathrm{p}=0.005)$ based on monthly income. There was a significant difference in acculturative stress $(\mathrm{F}=4.41, \mathrm{p}=0.005)$, depression $(\mathrm{F}=5.52, \mathrm{p}<0.001)$, and $\mathrm{QOL}(\mathrm{F}=9.49, \mathrm{p}<0.001)$ based on the number of comorbidities.

\begin{tabular}{|c|c|c|c|c|c|c|c|}
\hline \multirow[b]{2}{*}{ Variables } & \multirow{2}{*}{ Classification } & \multicolumn{2}{|c|}{ Acculturative stress } & \multicolumn{2}{|l|}{ Depression } & \multicolumn{2}{|l|}{ Quality of life } \\
\hline & & $\begin{array}{l}\text { Mean } \quad \pm \\
\text { (SD) }\end{array}$ & $t$ or $F$ & Mean \pm SD & $t$ or $F$ & Mean \pm SD & $t$ or $F$ \\
\hline \multirow{6}{*}{$\begin{array}{l}\text { Age(years) } \\
(M \pm S D 52.88 \pm 1.01)\end{array}$} & $20-29$ & $\begin{array}{ll}89.69 & \pm \\
16.59 & \end{array}$ & \multirow{6}{*}{$1.69(0.138)$} & $\begin{array}{ll}9.85 & \pm \\
12.12 & \end{array}$ & \multirow{6}{*}{$1.29(.268)$} & $\begin{array}{ll}149.46 & \pm \\
17.36 & \end{array}$ & \multirow{6}{*}{$0.68(.636)$} \\
\hline & $30-39$ & $\begin{array}{ll}96.00 \\
15.26\end{array}$ & & $\begin{array}{l}15.91 \\
13.98\end{array}$ & & $\begin{array}{ll}143.94 & \pm \\
22.59 & \end{array}$ & \\
\hline & $40-49$ & $\begin{array}{ll}94.79 & \pm \\
10.48 & \end{array}$ & & $\begin{array}{ll}13.17 & \pm \\
11.69 & \end{array}$ & & $\begin{array}{ll}142.17 & \pm \\
22.82 & \end{array}$ & \\
\hline & $50-59$ & $98.40 \pm 8.98$ & & $\begin{array}{ll}17.81 & \pm \\
16.11 & \end{array}$ & & $\begin{array}{ll}140.67 & \pm \\
25.04 & \end{array}$ & \\
\hline & $60-69$ & $\begin{array}{ll}96.94 & \pm \\
11.66 & \end{array}$ & & $\begin{array}{ll}18.41 \\
14.52)\end{array}$ & & $\begin{array}{ll}136.34 & \pm \\
27.62 & \end{array}$ & \\
\hline & $\geq 70$ & $\begin{array}{l}92.48 \\
12.67\end{array}$ & & $\begin{array}{ll}15.23 & \pm \\
13.83 & \end{array}$ & & $\begin{array}{ll}143.40 & \pm \\
28.18 & \end{array}$ & \\
\hline \multirow{2}{*}{ Gender } & Male & $\begin{array}{l}97.60 \\
13.46\end{array}$ & \multirow{2}{*}{$1.87(0.063)$} & $\begin{array}{ll}15.72 & \pm \\
14.20 & \end{array}$ & \multirow{2}{*}{$0.20(.837)$} & $\begin{array}{ll}137.35 & \pm \\
25.99 & \end{array}$ & \multirow{2}{*}{$-1.76(.837)$} \\
\hline & Female & $\begin{array}{l}94.16 \quad \pm \\
12.26\end{array}$ & & $\begin{array}{ll}15.31 & \pm \\
13.76 & \end{array}$ & & $\begin{array}{ll}143.75 & \pm \\
24.24 & \end{array}$ & \\
\hline \multirow{2}{*}{ Marital status } & Unmarried & $\begin{array}{l}95.00 \\
15.57\end{array}$ & \multirow{2}{*}{$0.07(0.975)$} & $\begin{array}{ll}15.06 & \pm \\
14.66 & \end{array}$ & \multirow{2}{*}{$0.83(.476)^{*}$} & $\begin{array}{ll}145.20 & \pm \\
18.16 & \end{array}$ & \multirow{2}{*}{$0.37(.773)$} \\
\hline & Married & $\begin{array}{l}95.35 \\
12.15\end{array}$ & & $\begin{array}{ll}16.11 & \pm \\
14.18 & \end{array}$ & & $\begin{array}{ll}140.71 & \pm \\
25.46 & \end{array}$ & \\
\hline
\end{tabular}


Citation: Hyun Oh J (2015) Relationships between Acculturative Stress, Depression, and Quality of Life on in North Korean Refugees Living in South Korea. J Health Edu Res Dev 3: 142. doi:10.4172/2380-5439.1000142

Page 5 of 8

\begin{tabular}{|c|c|c|c|c|c|c|c|}
\hline & Divorced & $\begin{array}{l}95.44 \\
11.47\end{array}$ & & $\begin{array}{ll}17.10 & \pm \\
15.23 & \end{array}$ & & $\begin{array}{ll}140.97 & \pm \\
33.68 & \end{array}$ & \\
\hline & Bereaved & $\begin{array}{ll}94.36 & \pm \\
12.74 & \end{array}$ & & $\begin{array}{ll}12.66 & \pm \\
11.16 & \end{array}$ & & $\begin{array}{ll}143.52 & \pm \\
21.15 & \end{array}$ & \\
\hline \multirow{2}{*}{ Living with } & Alone & $\begin{array}{ll}96.07 & \pm \\
12.18 & \end{array}$ & \multirow{2}{*}{$0.88(0.378)$} & $\begin{array}{ll}16.86 & \pm \\
14.12 & \end{array}$ & \multirow{2}{*}{$1.22(.221)^{\star}$} & $\begin{array}{ll}140.93 & \pm \\
27.55 & \end{array}$ & \multirow{2}{*}{$-0.48(.627)$} \\
\hline & Family & $\begin{array}{l}94.55 \\
12.96\end{array}$ & & $\begin{array}{ll}15.56 & \pm \\
13.68 & \end{array}$ & & $\begin{array}{ll}142.57 & \pm \\
23.16 & \end{array}$ & \\
\hline \multirow{4}{*}{ Religion } & Protestant & $\begin{array}{l}94.32 \\
12.89\end{array}$ & \multirow{4}{*}{$2.36(0.072)$} & $\begin{array}{ll}15.35 & \pm \\
14.11 & \end{array}$ & \multirow{4}{*}{$2.52(.059)$} & $\begin{array}{ll}142.87 & \pm \\
25.39 & \end{array}$ & \multirow{4}{*}{$1.84(.141)$} \\
\hline & Catholic & $\begin{array}{l}102.50 \quad \pm \\
2.12\end{array}$ & & $1.00 \pm 1.41$ & & $156.50 \pm 2.12$ & \\
\hline & Buddhist & $\begin{array}{l}89.90 \\
12.24\end{array}$ & & $7.10 \pm 9.15$ & & $\begin{array}{ll}152.40 \\
19.96\end{array}$ & \\
\hline & None & $\begin{array}{l}98.69 \\
12.67\end{array}$ & & $\begin{array}{ll}17.96 & \pm \\
13.17 & \end{array}$ & & $\begin{array}{ll}136.04 & \pm \\
23.30 & \end{array}$ & \\
\hline \multirow{4}{*}{ Education } & None & $98.00 \pm 2.83$ & \multirow{4}{*}{$5.09(0.002) \dagger$} & $8.00 \pm 5.66$ & \multirow{4}{*}{$1.08(.355)$} & $\begin{array}{ll}133.00 & \pm \\
11.31 & \end{array}$ & \multirow{4}{*}{$0.96(.409)$} \\
\hline & Elementary & $\begin{array}{l}85.50 \\
16.15\end{array}$ & & $\begin{array}{ll}13.90 & \pm \\
16.29 & \end{array}$ & & $\begin{array}{l}153.40 \quad \pm \\
36.55\end{array}$ & \\
\hline & Middle-high & $\begin{array}{l}97.51 \\
12.02\end{array}$ & & $\begin{array}{ll}16.75 & \pm \\
14.68 & \end{array}$ & & $\begin{array}{ll}140.61 & \pm \\
26.58 & \end{array}$ & \\
\hline & College & $\begin{array}{l}92.44 \quad \pm \\
12.46\end{array}$ & & $\begin{array}{ll}13.70 & \pm \\
12.20 & \end{array}$ & & $\begin{array}{ll}142.93 & \pm \\
20.22 & \end{array}$ & \\
\hline \multirow{3}{*}{ Economic status } & Higha & $87.50 \pm 2.12$ & \multirow{3}{*}{$2.72(0.068)$} & $\begin{array}{ll}15.50 & \pm \\
2.12 & \end{array}$ & \multirow{3}{*}{$0.70(.494)$} & $147.00 \pm 4.24$ & \multirow{3}{*}{$\begin{array}{l}6.09(0.003) \dagger \\
b>a, c\end{array}$} \\
\hline & Middleb & $\begin{array}{l}90.36 \\
10.35\end{array}$ & & $\begin{array}{l}12.50 \\
14.19\end{array}$ & & $\begin{array}{ll}156.89 & \pm \\
12.69 & \end{array}$ & \\
\hline & Lowc & $\begin{array}{ll}95.86 & \pm \\
12.88 & \end{array}$ & & $\begin{array}{ll}15.83 & \pm \\
13.87 & \end{array}$ & & $\begin{array}{ll}139.83 & \pm \\
25.53 & \end{array}$ & \\
\hline \multirow{2}{*}{ Smoking } & Yes & $\begin{array}{ll}96.58 & \pm \\
14.15 & \end{array}$ & \multirow{2}{*}{$-0.68(.492)$} & $\begin{array}{ll}17.84 & \pm \\
14.43 & \end{array}$ & \multirow{2}{*}{$-1.04(.298)^{\star}$} & $\begin{array}{ll}135.87 & \pm \\
30.99 & \end{array}$ & \multirow{2}{*}{$1.46(.143)$} \\
\hline & No & $\begin{array}{l}94.90 \\
12.45\end{array}$ & & $\begin{array}{l}15.05 \\
13.77\end{array}$ & & $23.72 \pm 1.67$ & \\
\hline \multirow{2}{*}{ Drinking } & Yes & $\begin{array}{l}94.97 \\
13.29\end{array}$ & \multirow{2}{*}{$0.12(.903)$} & $\begin{array}{l}13.81 \\
13.15\end{array}$ & \multirow{2}{*}{$1.22(.222)$} & $\begin{array}{l}140.63 \quad \pm \\
28.30\end{array}$ & \multirow{2}{*}{$0.56(.574)$} \\
\hline & No & $\begin{array}{l}95.19 \quad \pm \\
12.40\end{array}$ & & $\begin{array}{l}13.81 \\
13.15\end{array}$ & & $\begin{array}{l}140.63 \quad \pm \\
28.30\end{array}$ & \\
\hline \multirow{2}{*}{ Exercise } & Yes & $\begin{array}{ll}93.68 & \pm \\
11.44 & \end{array}$ & \multirow{2}{*}{$2.09(.037)^{\star}$} & $\begin{array}{l}12.41 \\
12.12\end{array}$ & \multirow{2}{*}{$4.11(<.001) \dagger$} & $\begin{array}{l}148.55 \quad \pm \\
19.83\end{array}$ & \multirow{2}{*}{$-5.11(<.001) \dagger$} \\
\hline & No & $\begin{array}{l}97.20 \\
14.06\end{array}$ & & $\begin{array}{l}19.77 \\
15.07\end{array}$ & & $\begin{array}{l}132.44 \quad \pm \\
28.17\end{array}$ & \\
\hline \multirow{2}{*}{ Occupation } & Yes & $\begin{array}{l}96.55 \\
12.30\end{array}$ & \multirow{2}{*}{$0.82(.408)$} & $\begin{array}{l}11.48 \\
11.69\end{array}$ & \multirow{2}{*}{$-2.11(.036)^{*}$} & $\begin{array}{l}143.50 \quad \pm \\
18.65\end{array}$ & \multirow{2}{*}{$.45(.648)$} \\
\hline & None & $\begin{array}{ll}94.79 & \pm \\
12.73 & \end{array}$ & & $\begin{array}{ll}16.35 \\
14.19\end{array}$ & & $\begin{array}{ll}141.60 \quad & \pm \\
26.12 & \end{array}$ & \\
\hline $\begin{array}{l}\text { Monthly income } \\
\text { (10,000won) }\end{array}$ & $\leq 50$ & $\begin{array}{l}96.72 \\
12.13\end{array}$ & $2.27(.080)$ & $\begin{array}{l}18.43 \\
14.93\end{array}$ & 4.32(.005)† & $\begin{array}{l}140.32 \quad \pm \\
26.17\end{array}$ & $.92(.431)$ \\
\hline
\end{tabular}


Citation: Hyun Oh J (2015) Relationships between Acculturative Stress, Depression, and Quality of Life on in North Korean Refugees Living in South Korea. J Health Edu Res Dev 3: 142. doi:10.4172/2380-5439.1000142

Page 6 of 8

\begin{tabular}{|c|c|c|c|c|c|c|c|}
\hline & $51-99 a$ & $\begin{array}{l}93.17 \quad \pm \\
12.68\end{array}$ & & $\begin{array}{l}12.79 \\
12.29\end{array}$ & & $\begin{array}{l}142.89 \\
23.58\end{array}$ & \\
\hline & $100-149 b$ & $\begin{array}{l}97.45 \quad \pm \\
14.11\end{array}$ & & $\begin{array}{l}14.68 \\
13.08\end{array}$ & & $\begin{array}{l}141.14 \quad \pm \\
26.41\end{array}$ & \\
\hline & $\geq 150$ & $\begin{array}{l}89.55 \\
12.96\end{array}$ & & $7.09 \pm 7.98$ & & $\begin{array}{l}152.91 \quad \pm \\
15.78\end{array}$ & \\
\hline \multirow{4}{*}{$\begin{array}{l}\text { Duration in } \\
\text { South Korea(years) }\end{array}$} & $1-5$ & $\begin{array}{l}92.85 \\
13.68\end{array}$ & \multirow{4}{*}{$1.53(.207)$} & $\begin{array}{l}16.07 \\
14.42\end{array}$ & \multirow{4}{*}{$1.91(.129)$} & $\begin{array}{ll}149.19 & \pm \\
27.93 & \end{array}$ & \multirow{4}{*}{ 1.59(.191) } \\
\hline & $6-10$ & $\begin{array}{l}94.85 \\
12.99\end{array}$ & & $\begin{array}{l}13.80 \\
12.73\end{array}$ & & $\begin{array}{ll}141.08 & \mathbf{I} \\
25.31 & \end{array}$ & \\
\hline & $11-15$ & $\begin{array}{l}97.92 \quad \pm \\
10.55\end{array}$ & & $\begin{array}{l}19.09 \\
15.64\end{array}$ & & $\begin{array}{l}138.39 \\
20.82\end{array}$ & \\
\hline & $\geq 16$ & $\begin{array}{l}90.25 \\
12.66\end{array}$ & & $\begin{array}{l}14.25 \\
14.86\end{array}$ & & $\begin{array}{l}144.00 \quad \pm \\
15.44\end{array}$ & \\
\hline \multirow{4}{*}{$\begin{array}{l}\text { Number } \\
\text { of comorbidity } \\
(\mathrm{M} \pm \mathrm{SD}, 1.05 \pm .80)\end{array}$} & $0 a$ & $\begin{array}{l}91.60 \quad \pm \\
12.27\end{array}$ & \multirow{4}{*}{$\begin{array}{l}4.41(0.005) \dagger \\
a<c\end{array}$} & $\begin{array}{l}10.68 \quad \pm \\
11.12\end{array}$ & \multirow{4}{*}{$\begin{array}{l}5.52(.001) \dagger \\
a<c\end{array}$} & $\begin{array}{ll}150.86 & \mathbf{I} \\
15.29 & \end{array}$ & \multirow{4}{*}{$\begin{array}{l}9.49(<.001) \dagger \\
a, b>c, d\end{array}$} \\
\hline & $1-2 b$ & $\begin{array}{l}95.55 \\
12.29\end{array}$ & & $\begin{array}{l}15.94 \\
14.10\end{array}$ & & $\begin{array}{ll}142.62 & \pm \\
25.54 & \end{array}$ & \\
\hline & $3-4 c$ & $\begin{array}{l}98.08 \\
12.99\end{array}$ & & $\begin{array}{l}21.28 \\
14.74\end{array}$ & & $\begin{array}{ll}126.33 & \pm \\
29.44 & \end{array}$ & \\
\hline & $\geq 5 d$ & $\begin{array}{l}107.00 \quad \pm \\
12.19\end{array}$ & & $\begin{array}{l}20.66 \quad \pm \\
15.94\end{array}$ & & $\begin{array}{l}128.33 \quad \pm \\
14.03\end{array}$ & \\
\hline
\end{tabular}

Table 3: Acculturative Stress, Depression, and QOL Scores by Participants’ Demographic Characteristics $(\mathrm{N}=232) .{ }^{*} \mathrm{p}<0.05, \dagger \mathrm{p}<0.01$.

\section{The correlations between acculturative stress, depression,} and QOL

Pearson coefficients are shown in Table 4. There was a moderate positive correlations between acculturative stress and depression $(\mathrm{r}=0.46, \mathrm{p}<0.001)$. There were moderate negative correlations between QOL and acculturative stress $(\mathrm{r}=-0.50, \mathrm{p}<0.001)$ and depression $(\mathrm{r}=-0.58, \mathrm{p}<0.001)($ Table 4$)$.

\begin{tabular}{|l|l|l|l|}
\hline & Acculturative stress & Depression & Quality of life \\
\hline Acculturative stress & 1 & & \\
\hline Depression & $0.46(<.001) \dagger$ & 1 & \\
\hline Quality of life & $-0.50(<.001) \dagger$ & $-.58(<.001) \dagger$ & 1 \\
\hline
\end{tabular}

Table 4: The Correlations between Acculturative Stress, Depression, and Quality of Life. ${ }^{*} \mathrm{p}<0.05, \dagger \mathrm{p}<0.01$.

\section{Factors that influence QOL}

A multiple regression analysis was conducted to identify important predictors of QOL among North Korean refugees. The results revealed that the most powerful predictor was depression. Depression, acculturative stress, exercise, and number of comorbidities account for $45.4 \%$ of the variance in QOL in North Korean refugees. The regression model revealed that depression $(\beta=-0.295)$ was a strong factor in predicting QOL (Table 5).

\begin{tabular}{|l|l|l|l|l|l|l|}
\hline Variables & $\boldsymbol{\beta}$ & $\mathbf{t}$ & $\mathbf{p}$ & $\begin{array}{l}\text { Adj. } \\
\mathbf{R}^{2}\end{array}$ & $\mathbf{F}$ & $\mathbf{p}$ \\
\hline
\end{tabular}

\begin{tabular}{|c|c|c|c|c|c|c|}
\hline (Constant) & & 9.97 & $<0.001$ & \multirow{17}{*}{0.454} & \multirow{17}{*}{10.455} & \multirow{17}{*}{$<0.001$} \\
\hline Age & 0.025 & 0.38 & 0.700 & & & \\
\hline Gender & 0.061 & 0.99 & 0.323 & & & \\
\hline Education & 0.020 & 0.34 & 0.727 & & & \\
\hline Marital status & -0.009 & -0.16 & 0.873 & & & \\
\hline Living with & -0.032 & -0.54 & 0.588 & & & \\
\hline Religion & 0.005 & 0.08 & .934 & & & \\
\hline Smoking & -0.029 & -0.48 & 0.628 & & & \\
\hline Drinking & -0.036 & -0.62 & 0.534 & & & \\
\hline Exercise & 0.216 & 3.38 & $<.001 \dagger$ & & & \\
\hline Job & -0.006 & -0.10 & 0.918 & & & \\
\hline Monthly income & -0.062 & -0.97 & 0.329 & & & \\
\hline Economic status & -0.104 & -1.86 & 0.064 & & & \\
\hline $\begin{array}{l}\text { Duration in South } \\
\text { Korea }\end{array}$ & -0.029 & -0.51 & 0.607 & & & \\
\hline $\begin{array}{l}\text { Length of stay in a } \\
\text { third country }\end{array}$ & 0.035 & 0.62 & 0.531 & & & \\
\hline $\begin{array}{l}\text { Number } \\
\text { of comorbidity }\end{array}$ & -0.170 & -2.74 & $0.007 \dagger$ & & & \\
\hline Acculturative stress & -0.294 & -4.90 & $<0.001 \dagger$ & & & \\
\hline
\end{tabular}


depression $-0.295$ $-4.78$ $<0.001 \dagger$

Table 5: Multiple Regression Analysis of Influencing Factors of Quality of Life $(\mathrm{N}=232) .{ }^{*} \mathrm{p}<0.05, \dagger \mathrm{p}<0.01$.

\section{Discussion}

The present data revealed that North Korean refugees in South Korea exhibit moderate acculturative stress, mild depression, and low QOL. This study elucidates the degree of acculturative stress, depression, and QOL in North Korean refugees. Participants had lived in South Korea for an average of 8.3 years $(\mathrm{SD}=2.52)$. Among North Korean refugees, depressive symptoms (BDI scores greater than 16) were found in $40.9 \%$ of the total sample, which is higher than previously reported [22]. The prevalence of depressive symptoms found in this study was higher than that found in Korean immigrants in the Eastern United States (30.0\% of the participants) [23]. Living in a foreign country, leaving without family, and being away from home alone, can cause nervousness and depression [24]. These data show that North Korean refugees are more troubled than Korean immigrants.

In this study, there was a significant difference in depression based on exercise, job, monthly income, and number of comorbidities. North Korean refugees who exercise have fewer depressive symptoms than those who do not exercise. North Korean refugees with a job have fewer depressive symptoms than those who do not have a job. North Korean refugees with a high monthly income fewer depressive symptom than those with a low monthly income. Previous research indicates that economic factors have influence depression $[16,25]$. Similarly, individuals with a lower family income report more depressive symptoms [26]. In addition, as depressive conditions increase, the prevalence of comorbidities increases.

North Korean refugees were exposed to poor medical services while they were escaping from North Korea and settling in South Korea. This likely caused psychological problems. To prevent depression at this early stage, North Korean refugees need economic and public health assistance. Similar to previous studies, this study revealed that unemployment and a subjective sense that one's health is poor were correlated with depressive symptoms in North Korean refugees [27].

Analysis of the correlation between BDI and acculturative stress in North Korean refugees revealed that higher BDI scores were associated with higher acculturative stress scores. The correlation between BDI and QOL revealed that higher BDI scores were associated with lower QOL, and the correlation between acculturative stress and QOL showed that higher acculturative stress was associated with lower QOL.

North Korean refugees, who feel they have low QOL, low psychological health, high acculturative stress, low physical health, low economic status, and low government funding experience greater depression.

In this study, acculturative stress was one of the most psychologically damaging factors. Acculturative stress was higher when depression was higher and QOL was lower. It is important to note that depression has been found to be a significant contributor to acculturative stress in other ethnic groups [28]. Depression is one of the most prevalent mental health problems among immigrants in the US., and is associated with acculturative stress. A similar situation was reported by Bauer and Priebe [29], who examined 122 East German refugees who, experienced anxious-depressive symptoms immediately after migrating to West Berlin. Acculturative stress scores in this sample were higher than in the Kim study [29], where the mean acculturative stress score was 2.81 , and other previous studies. This difference may be due to the fact that our participants faced harsh conditions that threatened their survival. Furthermore, after settling in South Korea, rivalry and acculturative stress caused stress and anxiety. The loneliness and alienation experienced by North Korean defectors exacerbated these psychological problems.

North Koreans living in South Korea did not have access to adequate medical services, and health problems combined with one or two comorbidities can cause chronic illness. North Korean defectors with these problems may also have mental health problems. In addition, health interventions were not available. These findings indicate that mental health programs should provide early interventions for North Korean defectors who are experiencing psychological problems such as depressive symptoms and acculturative stress that could detract from their QOL and their adaptation to South Korean society.

There are some limitations to this study. Our sample may have been biased. It is unclear whether this sample would is representative of the all North Korean refugees living in South Korea. However, this study is valuable in that it serves as a foundation for assessing acculturative stress, depression, and QOL in North Korean refugees.

\section{Conclusion}

In conclusion, acculturative stress and depression impact QOL. It is important to focus on North Korean refugees to better understand the factors that contribute to lower QOL and to distinguish the effects of these factors. This study showed that lower QOL causes acculturative stress, depression, and physical comorbidities. This study also showed that economic status and monthly income influenced psychological status and QOL of North Korean refugees, and elucidated the mental health status of North Korean refugees living in South Korea. Therefore, special attention to QOL and psychological status in North Korean refugees is needed. In addition, mental and economic support should be provided to improve the psychiatric characteristics and QOL of North Korean refugees.

\section{References}

1. North Korean Refugees Foundation (2013) North Korean Refugees Immigration Status. Open Society foundation.

2. Ko SH, chung KS, Oh YS (2004) North korean defectors: their life and well-being after defection. Asian Perspective 28: 65-99.

3. Ministry of Unification (2003) Republic of Korea. Data for inspection of the ministry of unification conducted by the National Assembly.

4. Lee KY (2000) Support program for North Korean defectors who reside in South Korea, Reunification Collection of Treatises. Research Institute of Korean Unification 18: 33-54.

5. Jeon WT, Min SG (1996) Psychological and adaptational problems of North Korean Defectors. Yonsei University Reunification Thesis Collection II. Seoul: Oreum Publishing Inc.

6. Lee JH (1997) Development of psychosocial adaptation program for North Korean defectors. Sung-Gok Collection of Treatises 28: 739-789.

7. Jeon WT (1997) A study on defector's social adaptation and self-identity. Korean Unification Studies 1: 109-167.

8. Rudmin F (2009) Constructs, measurements and models of acculturation and acculturative stress. International Journal of Intercultural Relations. 33: $106-123$. 
Citation: Hyun Oh J (2015) Relationships between Acculturative Stress, Depression, and Quality of Life on in North Korean Refugees Living in South Korea. J Health Edu Res Dev 3: 142. doi:10.4172/2380-5439.1000142

Page 8 of 8

9. Berry JW (1997) Immigration, acculturation, and adaptation. International Review of Applied Psychology 46: 55-68.

10. Furnham A, Bochner S (1986) Culture shock, psychological reaction to unfamiliar environment. London: Great Britain Methen.

11. Jeon WT (1997) Review of adaptation and mental health of refugees and perspectives and counterplots in Korean reunification process. Journal of Korean Neuropsychiatric Association 36: 3-18.

12. Vonnahme LA and Lankau EW (2015) Factors Associated with Symptoms of Depression Among Bhutanese Refugees in the United States. J Immigr Minor Health 17: 1705-1714.

13. Roh DK (2001) The influences of individual differences on adjustment and mental health in North Korean Refugees. Seoul Chung-Ang University.

14. Jeon WT, Min SK, Lee MH, Lee ES (1997) Adjustment of North Korean defectors in South Korea. Journal of Korean Neuropsychiatric Association 36: 145-161.

15. Eom TW, Lee KY (2004) The relationship among social problem solving capability, social support and depression of North Korean defectors. Mental Health \& Social Work. 18: 5-32.

16. Jeon W, Hong C, Lee C, Kim DK, Han M, et al. (2005) Correlation between traumatic events and posttraumatic stress disorder among North Korean defectors in South Korea. Journal of Trauma Stress. 18:147-154.

17. Sandhu DS, Asrabadi BR (1994) Development of an Acculturative Stress Scale for International Students: preliminary findings. Psychol Rep 75 435-448.

18. Lee SJ (1996) Relations between the Acculturative Stress and Belief System, Social Support of International Student. The Journal of Yonsei Educational Research 9: 157-176.

19. Beck AT, Steer R, Brown G (1996) Beck depression inventory manual (2nd Edn) Psychological Corporation, San Antonio.

20. Kim MS, Lee IM, Lee CS (2007) The validation study of Korean BDI: In female university students sample. The Korean Journal of Clinical Psychology 26: 997-1014.
21. Ro YJ A (1988) Analytical study of the quality of life of the middle-aged in Seoul. Seoul: Younsei University Press.

22. Choi SJ (2005) Study of religious activities and depression scale in North Korean defectors. Seoul: Younsei University Press.

23. Kunkel EJ, Kim JS, Hann HW, Oyesanmi O, Menefee LA, et al. (2000) Depression in Korean immigrants with hepatitis B and related liver diseases. Psychosomatics 41: 472-480.

24. Chae SM, Park JW, Kang HS (2014) Relationships of acculturative stress, depression, and social support to health-related quality of life in vietnamese immigrant women in South Korea. Journal of transcultural nursing 25: 137-144.

25. Lee HY, Moon A, Knight BG (2004) Depression among elderly Korean immigrants: Exploring socio-cultural factors. Journal of Ethics \& Cultural diversity in Social Work 13:1-26.

26. Jeon BH, Kim MD, Hong SC, Kim NR, Lee CI, et al. (2009) Prevalence and correlates of depressive symptoms among North Korean defectors living in South Korea for more than one year. Psychiatry investigation 6: $122-130$

27. Ayers JW, Hofstetter RC, Usita P, Irvin VL, Kang S, et al. (2009) Sorting out the competing effects of acculturation, immigrant stress, and social support on depression: A report on Korean women in California. The Journal of Nervous and Mental Disease, 2009; 197: 742-747.

28. Bauer M1, Priebe S (1994) Psychopathology and long-term adjustment after crises in refugees from East Germany. Int J Soc Psychiatry 40: 165-176.

29. Kim CD (2012) The effect of perceived stress and self-esteem on depression among North Korean refugees: focusing on the moderating effect of discrimination and suppressive coping style. Seoul, Seoul National University. 